\title{
Tuberculosis congénita: presentación de un caso infrecuente
}

\author{
Congenital tuberculosis: presentation of a rare case
}

Prof. Asist. Dr. Velat Şen, ${ }^{a}$ Prof. Asist. Dra. Hadice Selimoğlu Şen, ${ }^{b}$ Prof. Asist. Dr. Fesih Aktar, ${ }^{c}$

Prof. Asist. Dr. Ünal Uluca, ${ }^{c}$ Prof. Asist. Dra. Müsemma Karabela y Prof. Dr. Mehmet Fuat Gürkan ${ }^{a}$

\begin{abstract}
RESUMEN
La tuberculosis congénita es una enfermedad rara, con alta tasa de mortalidad. Es considerada el resultado de la transmisión vertical de la infección desde la placenta al feto, a través de la aspiración de líquido amniótico o por vía transplacentaria a través de las venas umbilicales. El diagnóstico de la enfermedad suele ser difícil porque los signos clínicos son inespecíficos. Se presenta el caso de un lactante varón de 48 días de vida, que fue internado por pérdida de peso, fiebre, tos, hemoptisis y dificultad respiratoria durante los últimos 20 días. Había recibido antibióticos de amplio espectro durante ese lapso, sin presentar mejoría. La radiografía de tórax mostró una consolidación con una lesión cavitaria en los campos medio y superior izquierdo. Se detectó Mycobacterium tuberculosis por reacción en cadena de la polimerasa en una muestra tomada por lavado broncoalveolar y, con ese hallazgo, se diagnosticó tuberculosis congénita. Se comenzó, entonces, el tratamiento con tuberculostáticos. El paciente falleció al $13^{\text {er }}$ día de tratamiento. En lactantes con pérdida de peso, fiebre, tos, hemoptisis y dificultad respiratoria, debiera considerarse la posibilidad de tuberculosis congénita.

Palabras clave: niño, tuberculosis congénita, mortalidad.
\end{abstract}

http:/ /dx.doi.org/10.5546/aap.2015.e101

\section{INTRODUCCIÓN}

La infección con bacilo de tuberculosis durante la vida intrauterina se denomina tuberculosis congénita. La Organización Mundial de la Salud ha informado que la tuberculosis causó la muerte de medio millón de mujeres y de, al menos, 64000 niños en 2010. ${ }^{1}$ La tuberculosis ha causado también la orfandad de cerca de 10 millones de niños. ${ }^{1}$

La mortalidad global por tuberculosis ha sido informada como del 3,1\% (525 muertes

\footnotetext{
a. Departmento de Pediatría Pulmonar.

b. Departmento de Pulmonología.

c. Departmento de Pediatría.

Dicle University Medical School, Diyarbakir, Turquía.
}

Correspondencia:

Prof. Asist. Dr. Velat Şen: drvelatsen@hotmail.com, velat.sen@dicle.edu.tr

Conflicto de intereses: ninguno que declarar.

Recibido: 16-7-2014

Aceptado: 22-9-2014 sobre 17148 casos) de acuerdo con el informe de situación de 2012 en Turquía. ${ }^{2}$ La tuberculosis congénita ha sido reportada con frecuencia en regiones con alta prevalencia de tuberculosis en adultos. La alta tasa de tuberculosis entre mujeres en edad fértil en países en desarrollo también aumenta el riesgo de tuberculosis congénita. Aunque la incidencia de tuberculosis congénita es baja, su tasa de mortalidad de hasta el 50\% da cuenta de su importancia., En este artículo, se presenta, por su rareza y sus interesantes hallazgos radiológicos, un caso de tuberculosis congénita acompañada de un absceso paraespinal.

\section{CASO CLÍNICO}

Un lactante varón de 48 días de vida fue internado en el hospital con síntomas de pérdida de peso, fiebre, tos, hemoptisis y dificultad respiratoria. Los síntomas comenzaron el día 28 de vida. Fue internado por sospecha de infección bacteriana y recibió antibióticos de amplio espectro en diferentes centros de salud durante 20 días. A pesar del uso prolongado de antibióticos, no mejoró y fue derivado a un hospital terciario universitario.

Los antecedentes del paciente revelaron que fue un recién nacido de término, de bajo peso para su edad gestacional (2600 g), saludable al nacer y que no recibió ninguna vacuna en aquel momento.

El período posnatal temprano transcurrió con normalidad, pero a los 28 días de vida, desarrolló disnea progresiva, fiebre, anorexia y pérdida de peso.

Al momento del ingreso a nuestro hospital, su temperatura era $38,6^{\circ} \mathrm{C}$; la frecuencia cardíaca era 164/minuto; y la frecuencia respiratoria, 64/ minuto. Presentaba taquipnea significativa, disnea y retracciones sub e intercostales. La auscultación torácica reveló rales y roncus bilaterales. No se detectó hepatoesplenomegalia. La radiografía de tórax mostró áreas de condensación con una lesión cavitaria en los campos medios e inferior izquierdos (Figura 1). El hemograma mostró un recuento de leucocitos de $23,1 \times 10^{9}$ cel. $/ \mathrm{mm}^{3}$ (neutrófilos: $36,8 \%$; linfocitos: $48,2 \%$; y monocitos: 
11,5\%); hemoglobina de 7,9 $\mathrm{g} / \mathrm{dl}$ y recuento de plaquetas de $601 \times 10^{9}$ cél. $/ \mathrm{mm}^{3}$. Los valores de proteína $C$ reactiva y de eritrosedimentación fueron de $7,7 \mathrm{mg} / \mathrm{dl}$ y $41 \mathrm{~mm} / \mathrm{h}$, respectivamente. Los niveles de electrolitos séricos, la función hepática y renal fueron normales. Los cultivos de sangre y orina fueron negativos para Mycobacterium tuberculosis (MT). La tomografía computarizada de tórax mostró áreas de condensación con broncograma aéreo en la parte posterior del lóbulo superior izquierdo y una cavitación de 2 $\mathrm{cm}$ de diámetro en esa misma área (Figura $2 \mathrm{~A}$, $B)$. Además, hay áreas de vidrio esmerilado en las regiones posteriores del lóbulo superior derecho. Múltiples imágenes septales con refuerzo periférico que alcanzan un espesor de $2 \mathrm{~cm}$ del lado derecho y de 1,5 cm del izquierdo sugieren la presencia de un absceso paraespinal sobre el área paravertebral derecha e izquierda de la última vértebra torácica (Figura 2 C-F). La ecografía abdominal fue normal. Los niveles totales de Ig G, A y M fueron normales, por lo que se excluyó una posible inmunodeficiencia.

Dada la falta de respuesta al tratamiento con antibióticos al quinto día, se pensó en la posibilidad de infección por tuberculosis y se tomaron muestras de aspirado gástrico para evaluar la presencia de bacilos ácidoalcohol resistentes (BAAR). Estas muestras fueron negativas para BAAR con tinción del Ziehl Nielsen. La evaluación broncoscópica no mostró lesiones endobronquiales. Se detectó M. tuberculosis con el método de reacción en cadena de la polimerasa en el material obtenido

FIGURA 1. Área de condensación, con una lesión cavitaria en la zona superior y media izquierda de la radiografía de tórax

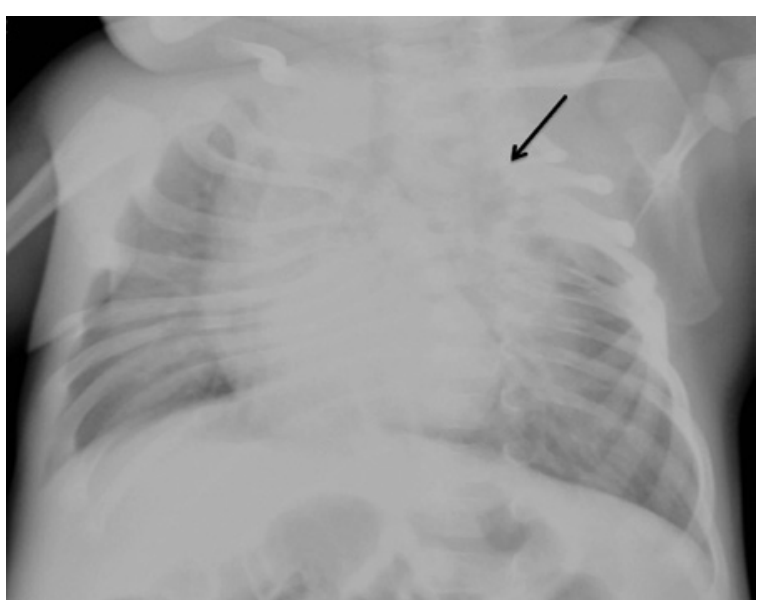

por lavado broncoalveolar al noveno día de internación. Se inició tratamiento con isoniazida, rifampicina y pirazinamida. Aunque se observó una leve mejoría clínica luego de siete días de tratamiento con tuberculostáticos, el paciente falleció el día 13 de tratamiento, luego de un episodio de hemoptisis masiva. El cultivo del material obtenido por lavado broncoalveolar fue informado como positivo para M. tuberculosis 40 días después de su obtención.

\section{Antecedentes maternos}

La madre del niño, de 31 años de edad, permaneció asintomática durante la gestación y el período posparto. Luego de la sospecha, se confirmó el diagnóstico de tuberculosis pulmonar y se inició el tratamiento. Refirió el contacto frecuente con una tía con antecedente de tuberculosis.

\section{COMENTARIO}

Hay dos vías principales de infección para tuberculosis congénita: puede ser transmitida por la madre por vía transplacentaria a través de la vena umbilical y formar un complejo primario en el hígado del niño con una diseminación hematógena secundaria. ${ }^{5} \mathrm{La}$ aspiración o ingestión de líquido amniótico que da lugar al complejo primario en el tracto gastrointestinal o los pulmones es la segunda vía de transmisión de la tuberculosis congénita. ${ }^{5}$ El hígado y el pulmón son los dos sitios más frecuentemente involucrados. Los signos y síntomas aparecen luego de la $3^{\text {a }}$ semana de vida a una edad mediana de 28 días. ${ }^{6}$

El diagnóstico de tuberculosis congénita debe considerarse en un niño con signos sugestivos de la enfermedad y una madre con historia de infección por $M$. tuberculosis o bien con signos sugestivos de esta. Sin embargo, hasta el $60-70 \%$ de las madres son asintomáticas y las manifestaciones en el neonato son, con frecuencia, inespecíficas. ${ }^{6-8}$ En un estudio reciente de Peng y col., se encontró que 162 madres tenían tuberculosis activa durante la gestación o posparto. De ellas, 121 sin historia de tuberculosis antes del embarazo fueron diagnosticadas luego de este. ${ }^{9}$ La madre del caso que se presenta estaba asintomática y fue diagnosticada con motivo de la búsqueda de la fuente de tuberculosis del niño. Los criterios para distinguir la tuberculosis congénita de aquella adquirida en la vida posnatal fueron establecidos inicialmente por Wallgren y, más tarde, revisados por Cantwell y col. en 
FIGURA 2. Tomografía computarizada de tórax, que revela áreas de condensación y cavitación en la zona posterior del lóbulo superior izquierdo (flechas en A y B). Absceso paraespinal en el área paravertebral al nivel de las vértebras torácicas inferiores (flechas en C-F)
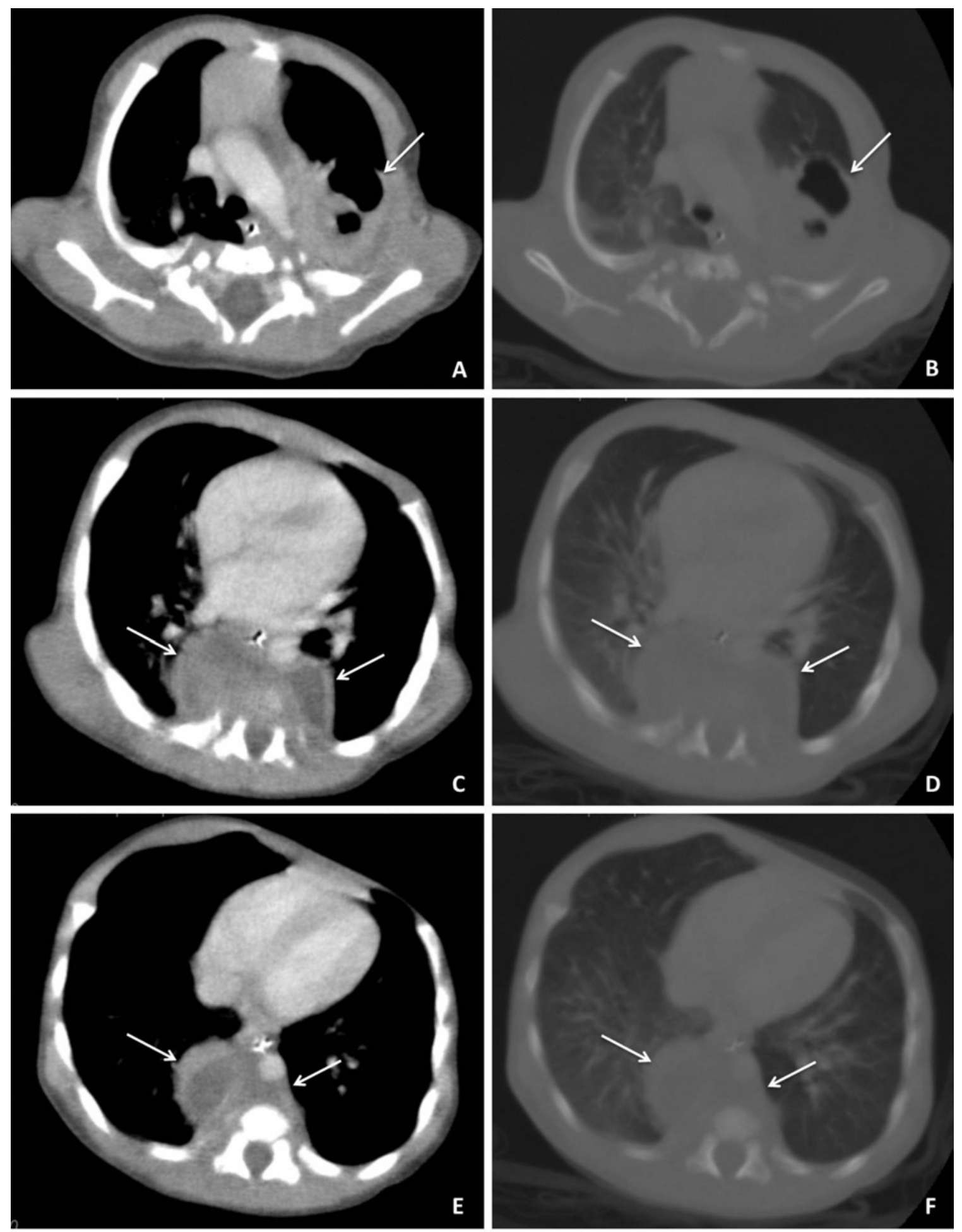
$1994^{6,10}$ (Tabla 1).

Los signos clínicos en el neonato infectado suelen ser inespecíficos. La neumonía, sepsis y meningitis purulenta causada por tuberculosis congénita son indistinguibles de las ocasionadas por otros gérmenes, por lo que es, con frecuencia, subdiagnosticada. ${ }^{9}$ La presencia de hepatoesplenomegalia y adenopatías puede guiar el diagnóstico, pero no ocurre en todos los casos. ${ }^{11}$

En nuestro caso, la ecografía abdominal normal sugiere que el foco primario fue el pulmón.

La tuberculosis congénita debiera sospecharse si los antibióticos resultan inefectivos y si las pruebas para otras infecciones congénitas son negativas, particularmente si la madre tiene antecedentes de tuberculosis o ha sido recientemente diagnosticada. Para el diagnóstico de tuberculosis, la prueba de tuberculina es positiva en menos del $15 \%$ de los casos de tuberculosis y puede tardar de uno a tres meses en volverse positiva. ${ }^{6,12}$ En nuestro caso, esta prueba fue negativa. El diagnóstico depende de la sospecha clínica y de la demostración de la presencia de BAAR en los tejidos o fluidos. En nuestro caso, pudo ser demostrado primero por la reacción en cadena de la polimerasa (PCR) y luego en cultivo de material de lavado broncoalveolar. No pudimos detectar el bacilo en muestras de orina y de sangre, posiblemente por no existir diseminación en el período posnatal.

La mayoría de los lactantes presentan una radiografía de tórax normal. Los hallazgos en las radiografías pueden variar desde las imágenes de neumonía intersticial, consolidación y cavitación a patrón miliar. En general, no es útil para realizar el diagnóstico. ${ }^{11}$ En la radiografía de nuestro paciente, había múltiples áreas de consolidación y lesiones cavitarias. También el paciente presentaba signos y síntomas inespecíficos, que remedaban los de la sepsis bacteriana. Solo un alto índice de sospecha luego del fracaso de los antibióticos de amplio espectro y de la exclusión de inmunodeficiencia llevó al diagnóstico.

Singh y col. informaron sobre hallazgos clínicos y de laboratorio que pueden sugerir tuberculosis congénita, los cuales incluyen a los recién nacidos de áreas endémicas para tuberculosis con neumonía de mala evolución sin respuesta al antibiótico, madre con tuberculosis y lactante con síntomas inespecíficos. ${ }^{13}$ Nuestro caso reunía estos tres criterios.

\section{CONCLUSIÓN}

Este caso ilustra la posibilidad de tuberculosis congénita en aquellos niños con infecciones que no responden favorablemente al tratamiento con antibióticos. Una historia clínica detallada de los pacientes es de especial utilidad en aquellas regiones con alta prevalencia de tuberculosis. En ausencia de antecedentes maternos, no debe excluirse este diagnóstico en los lactantes. La enfermedad puede ser subdiagnosticada debido a su amplia variabilidad clínica. Las formas congénitas y perinatales de esta enfermedad deberían ser consideradas, particularmente en los países en desarrollo, en los que hay alta prevalencia de tuberculosis.

TABLA 1. Criterios diagnósticos para tuberculosis congénita

\section{Criterios de Wallgren}

a. Lesión primaria localizada en el hígado o en nódulos linfáticos satélites correspondientes al sistema porta.

b. El pulmón es el sitio primario de infección (aspiración) y se está seguro de que la infección no es posnatal.

Criterios de Cantwell

Criterio primario: Debe probarse que el lactante tiene lesiones tuberculosas.

Criterios secundarios:

1. Lesiones en la primera semana de vida.

2. Complejo primario hepático o granulomas caseosos hepáticos.

3. Infección tuberculosa de la placenta o del tracto genital materno.

4. Exclusión de la posibilidad de la transmisión posnatal a través de la investigación de los contactos, que incluyen a quienes lo asistieron en el hospital, y por la adherencia a las recomendaciones existentes para tratar a los lactantes expuestos a tuberculosis. 


\section{Agradecimientos}

Agradecemos al Dr. Abdurrahim Dusak del Departamento de Radiología de la Dicle University Medical School por su contribución con las imágenes radiológicas.

\section{REFERENCIAS}

1. World Health Organization. Global tuberculosis report 2012. Geneva:World Health Organization;2012. Disponible en:http://www.who.int/tb/publications/global_report/ gtbr12_main.pdf. [Consulta: 24 de septiembre de 2014].

2. Ministry of Health of Turkey. 2012 Report on situation of tuberculosis in Turkey: Analysis of Treatment Results:89-102. Ankara: Ministry of Health of Turkey; 2013. Disponible en: Türkiye'de Verem Savaş Raporları. [Consulta: 24 de septiembre de 2014].

3. Crockett M, King SM, Kitai I, Jamieson F, et al. Nosocomial transmission of congenital tuberculosis in a neonatal intensive care unit. Clin Infect Dis 2004;39(11):1719-23.

4. Starke JR. Tuberculosis. An old disease but a new threat to the mother, fetus, and neonate. Clin Perinatol 1997;24(1):107-27.
5. HagemenJ,ShulmanS, Schreiber M,LuckS, etal.Congenital tuberculosis: critical reappraisal of clinical findings and diagnostic procedures. Pediatrics 1980;66(6):980-4.

6. Cantwell MF, Sehab ZM, Costello AM, Sands L, et al. Brief report: congenital tuberculosis. $N$ Engl J Med 1994;330(15):1051-4.

7. Hassan G, Qureshi W, Kadri SM. Congenital tuberculosis. JK Sci J Med Educ Res 2006;8:193-4.

8. Adhikari M, Pillay T, Pillay DG. Tuberculosis in the newborn: an emerging disease. Pediatr Infect Dis J 1997;16(12):1108-12.

9. Peng W, Yang J, Liu E. Analysis of 170 cases of congenital TB reported in the literature between 1946 and 2009. Pediatr Pulmonol 2011;46(12):1215-24.

10. Wallgren AJ. Tratado de tuberculosis infantil. Buenos Aires: El Ateneo; 1940:27-9.

11. Pillay T, Adhikari M. Congenital tuberculosis in a neonatal intensive care. Clin Infect Dis 1999;29(2):467-8.

12. Ormerod P. Tuberculosis in pregnancy and the puerperium. Thorax 2001;56(6):494-9.

13. Singh M, Kothur K, Dayal D, Kusuma S. Perinatal tuberculosis: a case series. J Trop Pediatr 2007;53(2):135-8. 